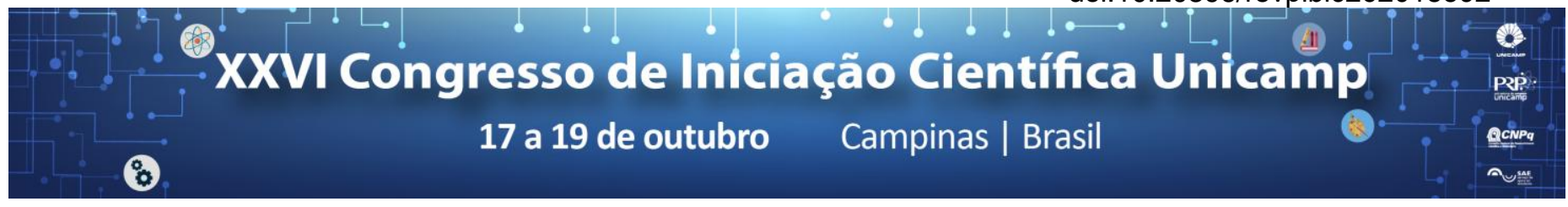

\title{
Body mass index and psychosocial stress in pharmacy undergraduate course
}

\author{
Heloísa Monteiro do Amaral-Prado*, Filipy Borghi, Camila Maiara Rocha-Teles, Samantha Mc Fadden, Camila \\ Lidiane de Morais, Carolina Silva, Priscila Cristina da Silva, Dora Maria Grassi-Kassisse.
}

\begin{abstract}
The literature suggests there may be a positive relationship between body mass gain and the psychosocial stress index (PSSI), which the emotional eating can be considered as an adaptive response to the chronic stress experienced by the individual. The university environment is considered a place of intense psychosocial stress, which can trigger changes in the eating habits of individuals. In this pilot study, volunteers from pharmacy undergraduate course, did not present any kind of correlation between Body Mass Index and high PSSI.
\end{abstract}

\section{Key words:}

Body mass index, psychosocial stress, undergraduate.

\section{Introduction}

Psychosocial stress is closely related to anxiety, depression, anger, apathy, and alienation ${ }^{1}$. Thereby, as the rewarding properties of food, the hyperpalatables, food with more fatty and rich in sugar and salt, can serve as comfort food. This acts as a form of self-medication to dispel the suffering experienced, a process called emotional eating ${ }^{1}$. In addition, studies suggest that psychosocial stress may lead to an increase in an individual's body mass (BMI) in relation to demographic and behavioral factors ${ }^{2,3}$. In this way, as the university environment is considered a local trigger of high psychosocial stress, due to all imposed demands and pressure, in this pilot study we aim to investigate the correlation between psychosocial stress index and BMI in pharmacy undergraduate students from Unicamp.

\section{Results and Discussion}

The project is approved by the Ethics Committee of Unicamp under the number CAAE 60640416.0.0000.5404. We calculated the BMI and scored the psychosocial stress questionnaires of 72 volunteers, 24 men and 48 women. All subjects are from pharmacy undergraduate course between de $1^{\text {st }}$ and $4^{\text {th }}$ year. The Sheldon-Cohen Psychosocial Stress Questionnaire was applied to investigate psychosocial stress, and BMI was calculated by weight and height measures, according to the formula BMI = weight $(\mathrm{kg}) /$ height $^{2}(\mathrm{~cm})$. The subjects exhibited the eutrophic profile (BMI 18.5-24.99), for both sexes, however they presented intermediate/high scores for PSSI (Figure 1). Thus, in this population there was no correlation between $\mathrm{BMI}$ and PSSI in the male and female population studied (Figure 2). We use GraphPad Prism 7 for statistical analyzes and figures. The normality of the data was investigated by the D'Agostino \& Pearson normality test and the correlation analysis performed was Spearman.
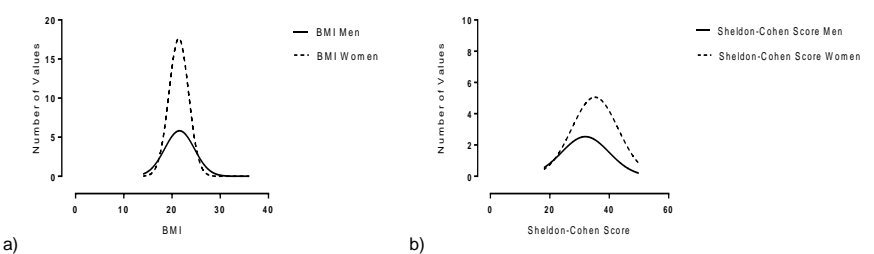

Figure 1. (A) Body mass index; (B) Sheldon Cohen psychosocial stress scores for volunteers men and women of pharmacy undergraduate course from Unicamp.
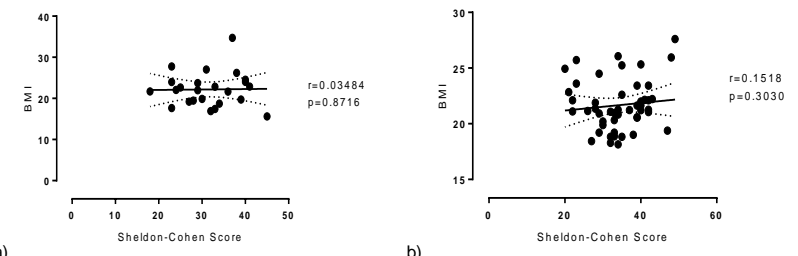

Figure 2. Correlation between Body Mass Index (BMI) and Psychosocial Stress (Sheldon-Cohen's instrument) for $(A)$ men and $(B)$ women of pharmacy undergraduate course from Unicamp. There was no direct correlation between the parameters in both sexes.

\section{Conclusions}

Although the intermediate/high PSSI scores, the volunteers of pharmacy undergraduate students showed to be eutrophic. Thus, in this population there was no correlation between BMI and PSSI.

\section{Acknowledgement}

All volunteers, PIBIC-CNPq and FAPESP.

${ }^{1}$ Yau YHC, Potenza MN. Stress and Eating Behaviors. Minerva Endocrinol. 2013; 38(3):255-67

${ }^{2}$ Lu Q, Hou F, Zhang Z, Tao F. Relationship between chronic psychosocial stress and BMI among adolescents. Zhonghua Liu Xing Bing Xue Za Zhi. 2016; 37(1):40-4.

${ }^{3}$ Harding JL, Backholer K, Williams ED, Peeters A, Cameron AJ, Hare MJ, et al. Psychosocial stress is positively associated with body mass index gain over 5 years: evidence from the longitudinal AusDiab study. Obesity (Silver Spring). 2014; 22(1):277-86. 